\title{
The Interior Temperature Distribution Measurement in a Rolling Tire
}

\author{
Mengxi Huang \\ Dept. of Modern Mechanics, \\ CAS Key Laboratory of Mechanical \\ Behavior and Design of Materials \\ University of Science and Technology \\ of China \\ Hefei, China, 230027
}

\author{
Ziran Li \\ Dept. of Modern Mechanics, \\ CAS Key Laboratory of Mechanical \\ Behavior and Design of Materials \\ University of Science and Technology \\ of China \\ Hefei, China, 230027 \\ lzr@mail.ustc.edu.cn
}

\author{
Yuanming Xia \\ Dept. of Modern Mechanics, \\ CAS Key Laboratory of Mechanical \\ Behavior and Design of Materials \\ University of Science and Technology \\ of China \\ Hefei, China, 230027
}

\begin{abstract}
A new wireless temperature measurement system which aims to measure the interior temperature distribution in a rolling tire is presented. In the system, Negative Temperature Coefficient (NTC) thermistor of bead type was selected as the temperature sensor; while a RF Module (Transmitters \& Receiver) was used to overcome the data transmission difficulty in rolling tires. Furthermore, to solve the unexpected sensor failure problem, two groups of thermistors, as substitutes for each other based on the hypothesis of uniform circumferential temperature, were placed at the same positions on two tire cross sections. The temperature distributions of both the inner wall and the tire cavity in the rolling tire were obtained. This effective and economical test method is able to obtain sufficient interior temperature information in rolling tires. The test results also confirm that there are no temperature gradients in tire circumferential direction.
\end{abstract}

Keywords-interior temperature; rolling tire; RF module; NTC thermistor; wireless data transmission

\section{INTRODUCTION}

Operating temperature is of vital concern to tire engineers because it relates to both endurance life and power loss of a tire. The heat sources which are mainly produced by the tire hysteresis losses contribute to the tire temperature rise. Several papers [1-5] have been presented on tire temperature prediction based on Finite Element Method. However, there are not enough measured temperatures to verify the predicted results, especially for the interior tire temperature.

The key points for the interior temperature measurement in rolling tires are sensors and data transmission methods. In the case of data transmission, when sensors are installed inside the tire, wireless data transmission is indispensable [6]. Although a slip ring can also be used in measurements for rotating tires [35, 7-8], abrasion of the brush and rings may occur and the slip ring does not have a long operational life.

The authors would like to thank Leilei Shang, Dongqing Hu and Professor Chao Zhai for excellent technical support on the design and installation of the temperature measurement system.
In terms of the temperature sensors, thermocouples and thermistors are widely used. Various authors [3-5, 7-8] have only measured the steady state temperatures at certain points in the tire body with buried thermocouples. However, more interior temperature information are needed, especially the tire cavity temperature distribution. Moreover, the sensor failure problems were reported in $[6,8]$.

To acquire sufficient interior temperature information in a rolling tire, a new wireless temperature measurement system was set up in this study. In the system, Negative Temperature Coefficient (NTC) thermistor of bead type was chosen as the temperature sensor due to its small size and economical cost; while a RF Module (Transmitters \& Receiver) was responsible for the data transmission from the interior of the tire to the external environment. In particular, to solve the unexpected sensor failure problem mentioned above, two groups of thermistors, as substitutes for one another based on the hypothesis of uniform circumferential temperature, were placed at the same positions on two tire cross sections. The temperature distributions of both the inner wall and the tire cavity in the rolling tire were obtained.

\section{The Wireless TeMPERATURE MEASUREMENT System}

The measurement system is illustrated in Fig. 1. The NTC thermistor is of MF52 type, with a bead diameter of about 2 $\mathrm{mm}$. Its Resistance-Temperature relationship is shown by the equation in Fig. 1, where $T_{0}$ is the reference temperature and $\mathrm{R}_{0}$ is the resistance at $\mathrm{T}_{0}$; $\mathrm{B}$ (Beta) is the material constant of the thermistor. The type of the RF Module (Transmitter \& Receiver) is Freescale MC13213. The thermistor links to the transmitter by wires, its resistance variations are converted to the digital voltage signals by the circuit and $\mathrm{A} / \mathrm{D}$ converter on the transmitter board, and then the signals are communicated from the transmitter to the receiver through radio at $2.4 \mathrm{GHz}$. The whole measurement system was calibrated with a Laboratory Thermostatic Water Bath. 


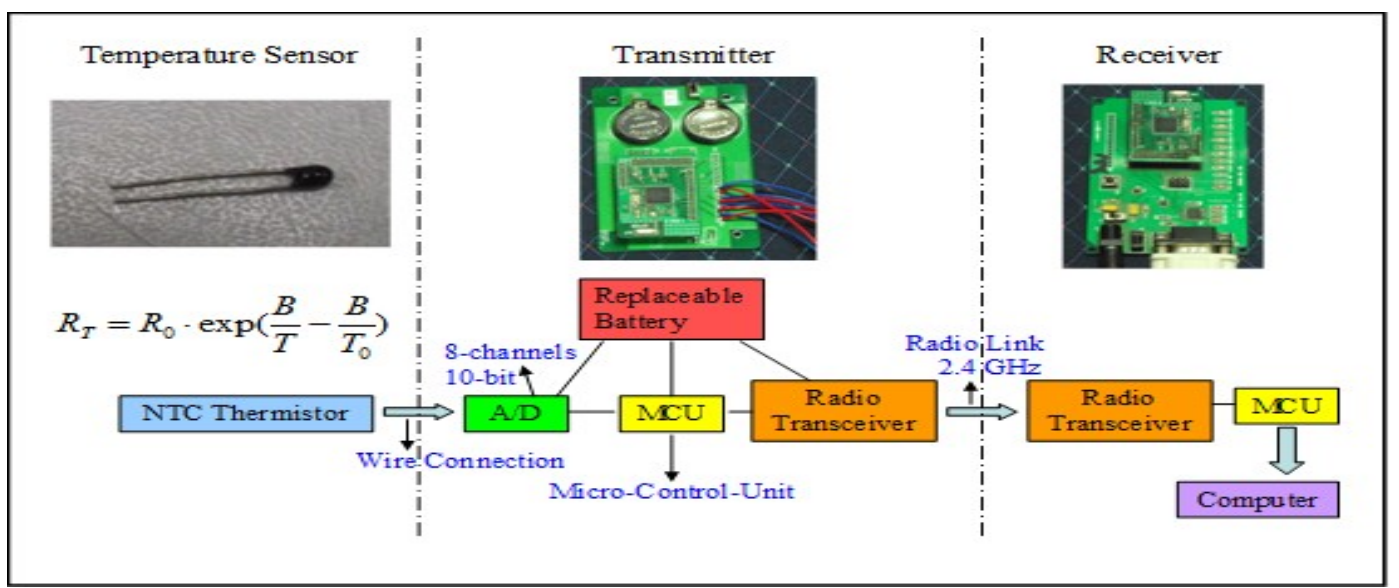

Figure1. Illustration of the Temperature Mesurement system

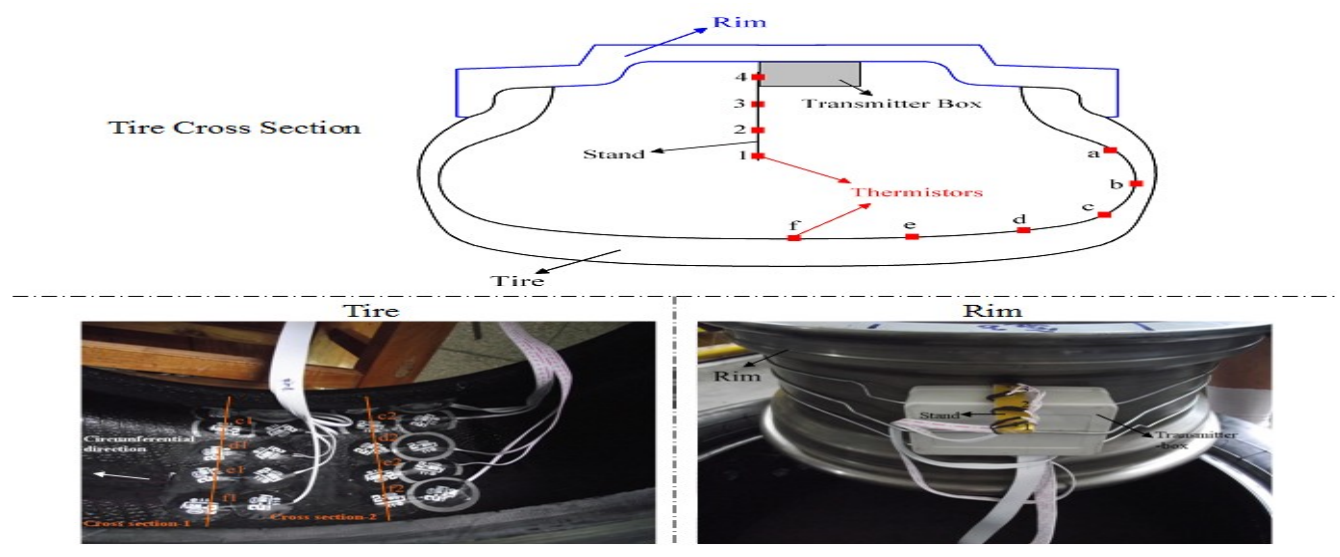

Figure 2. The System Mounting inside the Tire.

\section{EXPERIMENTAL}

The tire used in this study is a 205/55R16 smooth tread tire, and it ran on a tire endurance testing machine.

\section{A. The System Mounting inside the Tire}

As illustrated in Fig. 2, taking into account the symmetry, six thermistors (a f) were fixed at one side of the tire inner wall by using the patching rubber and the tire repair glue. The other four thermistors (1 4) that aim to measure the tire cavity temperatures were placed on the stand which was screwed to the transmitter box. The box contains two transmitter boards, and was bound on the rim by iron wire.

The reason why the sensors inside the tire body are vulnerable to failure is that they deform periodically with the tire, especially in the areas with big curvature. Based on the hypothesis of uniform circumferential temperature, we placed two groups of thermistors which are the substitutes for one another at the same positions on two tire cross sections. As long as each one of the two thermistors is functional throughout the testing, the target temperature can be obtained.

\section{B. Operating Conditions}

- Initial inflation pressure: $250 \mathrm{kPa}$

- Load: $5680 \mathrm{~N}$

- Speed: $60 \mathrm{~km} / \mathrm{h}$

- Ambient temperature: 302.5 K (Monitored by several mercury thermometers)

\section{RESUlts AND Discussion}

The temperatures of the inner wall and the contained air in the rolling tire are shown in Fig. 3 and Fig. 4. The curves demonstrate common features, which are described as follows. At the beginning of rolling, the tire temperature is in equilibrium with the ambient temperature. During unsteady state, the tire temperature rises due to the energy absorption from the heat source. After about forty minutes from the start, the tire arrives at a steady state when all heat generation in the tire is transferred to ambient air and road through the tire boundary.

As shown in Fig. 3, thermistors at various positions (b, d, e and f) are functional throughout the testing. The results 
confirm that there are no temperature gradients in tire circumferential direction. However, unexpected sensor failures occurred at position-a on cross section- 1 and position-c on cross section-2 respectively, which may attribute to some unexpected damages created during the assembly process,

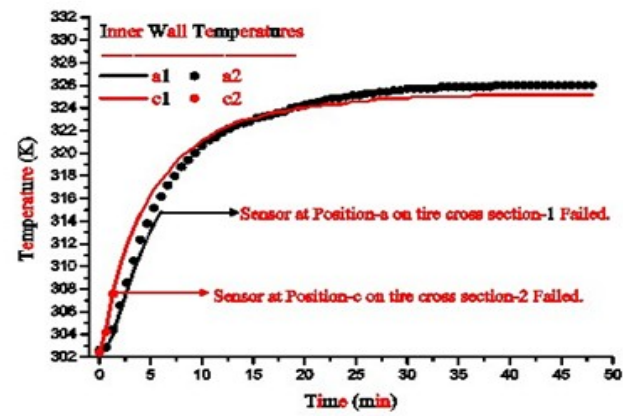

some unknown interactions inside the tire body during the testing, etc. But anyway, the thermistors at position-a

and position-c on the other cross section are functional so that we can still obtain the target temperatures.

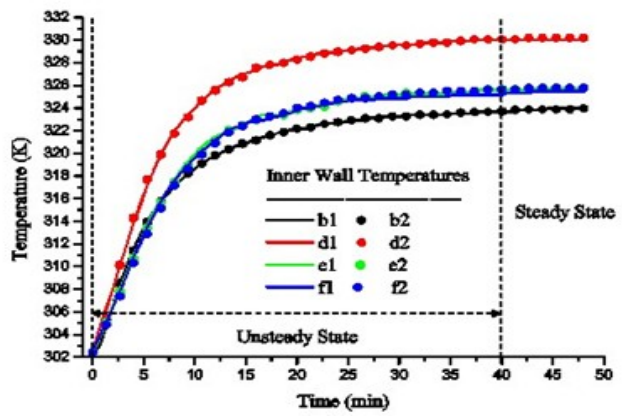

Figure 3. Inner Wall Temperatures in the Rolling Tire.

The four sensors (1 4) located in the tire cavity for contained air temperature measurement operates normally because they don't undergo the periodical deformation.

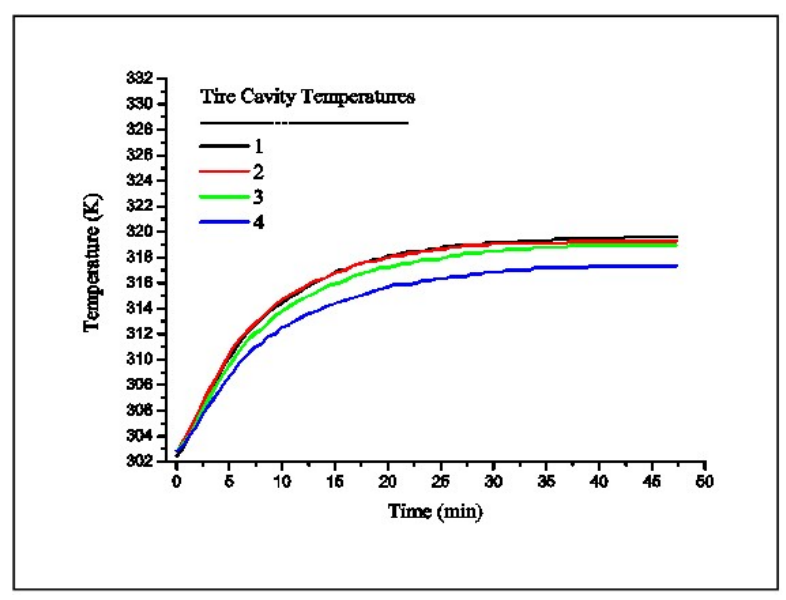

Figure 4.Contained Air Temperatures in the Rolling Tire.

\section{CONCLUSIONS}

In summary, we propose an effective and economical measurement method to obtain sufficient interior temperature information in rolling tires. The main advantages of this method are:

- Using wireless technology to overcome the data transmission difficulty inside the rolling tire.

- Taking advantage of the fact that there are no temperature gradients in tire circumferential direction (this has been confirmed in this paper) to solve the unexpected sensor failure problem.

It must be pointed out that a few unexpected sensor failures also occurred in this study. More flexible probes and wires may be the direction of further improvement.

\section{REFERENCES}

[1] K. Sarkar, Y. D. Kwon, and D. C. Prevorsek, “A New Approach to the Thermomechanical Analysis of Tires by Finite Element Method,” Tire Science and Technology, TSTCA, vol. 15, No. 4, pp. 261-275, 1987.

[2] B. Yavari, W. W. Tworzydlo, and J. M. Bass, “A Thermomechanical Model to Predict the Temperature Distribution of Steady State Rolling Tires,” Tire Science and Technology, TSTCA, vol. 21, No. 3, pp. 163168, 1993.

[3] B. S. Oh, Y. N. Kim, N. J. Kim, H. Y. Moon and H. Y. Park, "Internal Temperature Distribution in a Rolling Tire," Tire Science and Technology, TSTCA, vol. 23, No. 1, pp. 11-25, January-March 1995.

[4] H. C. Park, S. K. Youn, T. S. Song, and N. J. Kim, “Analysis of Temperature Distribution in a Rolling Tire Due to Strain Energy Dissipation,” Tire Science and Technology, TSTCA, vol. 25, No. 3, pp. 214-228, July-September 1997.

[5] T. G. Ebbott, R. L. Hohman, J. P. Jeusette and V. Kerchman, “Tire Temperature and Rolling Resistance Prediction with Finite Element Analysis,” Tire Science and Technology, TSTCA, vol. 27, No. 1, pp. 221, January-March 1999.

[6] R. Matsuzaki, A. Todoroki, "Wireless Monitoring of Automobile Tires for Intelligent Tires,” Sensors, vol. 8, pp. 8123-8138, December 2008.

[7] F. S. Conant, "Tire Temperatures,” Rubber Chemistry and Technology, vol. 44, No. 2, pp. 397-439, 1971.

[8] T. J. LaClair, C. Zarak, "Truck Tire Operating Temperatures on Flat and Curved Test Surfaces,” Tire Science and Technology, TSTCA, vol. 33, No.3,pp.156-178,July-September2005. 